\title{
A first list of vespid wasps from Vietnam (Hymenoptera: Vespidae)
}

\section{Первый список скмадчатокрылых ос (Hymenoptera: Vespidae) Вьетнама}

\author{
Phong Huy Pham ${ }^{1} \&$ Tingjing $\mathrm{Li}^{2}$ \\ Фонг Хай Пам ${ }^{1}$, Тинджинг $\Lambda u^{2}$
}

\begin{abstract}
${ }^{1}$ Institute of Ecology and Biological Resources, Vietnam Academy of Science and Technology, 18 Hoang Quoc Viet Road, Hanoi, Vietnam; e-mail: phong.wasp@gmail.com

${ }^{2}$ Institute of Entomology \& Molecular Biology, College of Life Sciences, Chongqing Normal University, Chongqing, 401331, P. R. China; e-mail: 1tjing1979@hotmail.com
\end{abstract}

KEY WORDS: Hymenoptera, Vespidae, Eumeninae, distribution, species list, Vietnam.

КЛЮЧЕВЫЕ СЛОВА: Нуmenoptera, Vespidae, Eumeninae, распространение, список видов, Вьетнам.

ABSTRACT: A first list of vespid wasps (Hymenoptera: Vespidae) recorded from Vietnam as of the end of 2013 is presented. A total of 109 species belonging to 32 genera of four subfamilies, including, Eumeninae (33 species), Stenogastrinae (10 species), Polistinae (50 species), and Vespinae (16 species), are treated as valid. 19 new distributional records and the information of the distribution of the species are also provided.

РЕЗЮМЕ: Приводится первый список складчатокрылых ос (Hymenoptera: Vespidae) Вьетнама. По состоянию на конец 2013 года он включает 109 видов из 32 родов четырёх подсемейств, в том числе Eumeninae (33 вида), Stenogastrinae (10 видов), Polistinae (50 видов) и Vespinae (16 видов). Для 19 видов приведены новые точки находок.

\section{Introduction}

The hymenopteran family Vespidae, with more than 5000 species worldwide, is divided into six subfamilies. They are Euparagiinae, Masarinae, Eumeninae, Stenogastrinae, Polistinae, and Vespinae with 10 species in 1 genus, 344 species in 14 genera, 3579 species in 210 genera, 58 species in 7 genera, 958 species in 26 genera, and 69 species in 4 genera, respectively [van der Vecht \& Carpenter, 1990, reviewed in Pickett \& Carpenter, 2010]. The social wasps are comprised of three subfamilies (Stenogastrinae, Polistinae, and Vespinae), in the family Vespidae, while the wasps in the three other subfamilies (Euparagiinae, Masarinae, and Eumeninae) are solitary.

South-East Asia is a special place for the wasps: it is the center of higher-level diversity. Four of the six vespid subfamilies occur (Eumeninae, Stenogastrinae, Polistinae, and Vespinae). The Eumeninae are cosmopolitan and include more than 3500 described species. The Stenogastrinae are endemic to the Indo-Malayan area, distributed from India and Sri Lanka to New Guinea. Five of the seven genera (Eustenogaster van der Vecht, 1969, Liostenogaster van der Vecht, 1969, Parischnogaster von Schulthess, 1914, Metischnogaster van der Vecht, 1977, and Cochlischnogaster Dong et Otsuka, 1997) occur in southern Asia, while the remaining two (Stenogaster Guerin, 1831 and Anischnogaster van der Vecht, 1972) are found only in New Guinea and its adjacent islands. The Polistinae are cosmopolitan, with two of the four tribes, Polistini and Ropalidiini, occurring in Old World. The Vespinae are Holarctic and Oriental, and Southeast Asia is the only area where all the four genera (Dolichovespula Rohwer, 1916, Vespula Thomson, 1869, Vespa Linnaeus, 1758, and Provespa Ashmead, 1903) occur, and Provespa is endemic to the area.

Vietnam, spread from north to south along the eastern margin of the Indochina Peninsula, covers a total area of approximately $331210 \mathrm{~km}^{2}$ with $4639 \mathrm{~km}$ long of the land boundary, and $3444 \mathrm{~km}$ long of the coastline. Vietnam's land is mostly hilly and densely forested. Mountains account for $40 \%$ of the country's land area, and tropical forests cover around $42 \%$. So Vietnam is expected to have high magnitude of biodiversity. Especially North Vietnam, which is within the Indo-Burma biodiversity hotspot and shows climatically more or less warm-temperate characteristics, is expected to be one of the centers of divergence of vespid wasps, as has been demonstrated for the polistine genus Polistes Latreille, 1802. Nevertheless, the vespid wasps in the area are yet poorly studied. An annotated distributional checklist of social wasps of Vietnam was performed by Nguyen et al. [2005]. In this checklist, they listed 45 species in 10 genera occurring in Vietnam. However, this work has hitherto been for 8 years, and many new species of the social wasp are added to the Vietnamese fauna since then. Specially, subfamily Eumeninae only treated formal in the middle of 2012. 
Well corroborated taxonomic works would of course contribute greatly to the study of the biology of these wasps, which exhibit considerable divergence in social organization and nesting behavior [reviewed in Turillazzi, 1991]. As the basis for perusing revisionary taxonomic works and catalogs, a present list provides basic taxonomic information and distribution records for wasps so far recorded from Vietnam.

\section{Materials and methods}

The present paper is based mainly on published faunistic records and also on specimens housed in Institute of Ecology and Biological Resources (IEBR), Hanoi, Vietnam. In each subfamily the genera are listed alphabetically. In each genus, species names are listed alphabetically insofar they are considered as valid in the most recent citation, and species are numbered, with a sub-number for each subspecies, if any. Distributional data are summarized by country for a taxon treated as valid. Provincial distribution and references are only for records from Vietnam. Vietnamese provinces are given in bold and given in alphabetical order in the lists. The specimens examined are listed by provinces in Vietnam. Because Ha Tay province merged into Hanoi capital 1 August 2008, the distribution of some species in this province is recorded for Hanoi. Abbreviations and symbols are as follows: National Park — NP; Phong Huy Pham - PP.

\section{Results and discussion}

Mainly based on previously published data and on specimens housed in IEBR, the present list of vespid wasps from Vietnam presented in this paper consists of 109 species in 32 genera of four subfamilies (Eumeninae, Stenogastrinae, Polistinae, and Vespinae). 19 new distributional records are added.

\section{Subfamily Eumeninae}

Allorhynchium van der Vecht, 1963

\section{Allorhynchium chinense (de Saussure, 1862)}

Rhynchium chinense de Saussure, 1862: 186; Allorhynchium chinense van der Vecht, 1963: 60; ?Allorhynchium argentatum Baltazar, 1966: 32.

DISTRIBUTION. China; Taiwan; Philippines; Vietnam [Giordani Soika, 1986].

NOTE. Giordani Soika [1986] recorded this species from Vietnam, but did not show exact locality.

\section{Antepipona de Saussure, 1855}

\section{Antepipona biguttata (Fabricius, 1787)}

Vespa biguttata Fabricius, 1787: 291; Antepipona injucunda Giordani Soika, 1973: 104; Antepipona biguttata biguttata Giordani Soika, 1981: 205, 207, 221; Antepipona biguttata takaoensis Giordani Soika, 1982: 207, 224

DISTRIBUTION. China: Hainan, Kwangtung, Kiangsi, Chekiang, Fukien, Yunnan; Taiwan; Thailand; Laos; Myanmar; Malaysia; India; Vietnam [Giordani Soika, 1982].

NOTE. Giordani Soika [1982] recorded this species from Vietnam, but did not show exact locality.
Anterhynchium de Saussure, 1863

3. Anterhynchium mellyi (de Saussure, 1852)

Rygchium mellyi de Saussure, 1852: 116; Rhynchium mellyi Saussure, 1855: 172; Odynerus mellyi Dover, 1929: 44; Anterhynchium mellyi van der Vecht, 1963: 76-77.

DISTRIBUTION. Allahabad; Bengal; Burma; China; Vietnam: Hoa Binh [van der Vecht, 1963].

Apodynerus Giordani Soika, 1993

4. Apodynerus formosensis continentalis Giordani Soika, 1994 217.

Apodynerus formosensis continentalis Giordani Soika, 1994:

DISTRIBUTION. China: Fukien, Guangdong, Kwangtung and Yen Ping; Laos; Vietnam: Vinh Phuc [Giordani Soika, 1994].

5. Apodynerus nitidiclypeus Gusenleitner, 2013

Apodynerus nitidiclypeus Gusenleitner, 2013: 121, 125. 2013].

DISTRIBUTION. Vietnam: Dac Lak [Gusenleitner,

Coeleumenes van der Vecht, 1963

6. Coeleumenes burmanicus (Bingham, 1897)

Montezumia burmanica Bingham, 1897: 349-350; Montezumia bisulcata Cameron, 1900: 535; Montezumia burmanica var. malayana Dover, 1931: 253; ?Montezumia (Pseudozumia) thoracica Sonan, 1939: 132; Pseudozumia burmanica Giordani Soika, 1941: 165; Pseudozumia burmanica var. malayana Giordani Soika, 1941: 168; Coeleumenes burmanicus van der Vecht, 1963: 46-47.

DISTRIBUTION. India: Sikkim, Assam, Siam; Malaysia; Vietnam: Tonkin, Than Moi [van der Vecht, 1963].

Delta Fabricius, 1775

\section{Delta campaniforme campaniforme}

(Fabricius, 1775)

Vespa campaniformis Fabricius, 1775: 371; Sphex campaniformis Christ, 1791: 312; Eumenes campaniformis Fabricius, 1804: 287; Eumenes esuriens F. Smith, 1861; Eumenes esuriens var. campaniformis Maindron, 1882: 270-272; Eumenes edwardsii von Schulthess, 1914: 263; Eumenes caffer var. gracilis Dover et Rao, 1922: 237; Eumenes gracilis var. esuriens Dover, 1926: 233; Eumenes campaniformis var. campaniformis Giordani Soika, 1934(1933): 227; Delta campaniforme campaniforme Giordani Soika, 1987: 147

DISTRIBUTION. Nepal; China; Thailand; Cambodia; Indonesia: Sumatra, Java, Moluccas, Papua; Papua New Guinea; Australia; Philippines; adventive in U. S. A.: Hawaii, Vietnam [de Saussure et al., 1904].

NOTE. de Saussure et al. [1904] recorded this species from Vietnam, but did not show exact locality.

\section{Delta esuriens (Fabricius, 1787)}

Vespa esuriens Fabricius, 1787: 293; Eumenes esuriens Fabricius, 1804: 286; Delta companiforme esuriens Krombein, 1991: 6; Delta esuriens Giordani Soika, 1992: 62.

DISTRIBUTION. India: Arunachal Pradesh, Delhi, Gujarat, Maharashtra, Orissa, Rajasthan, Tamil Nadu, Uttarakhand, West Bengal; Arabia; Indonesia: Borneo, Java; Iran; Iraq; Myanmar; Pakistan; Philippines; Sri Lanka; Thailand; Vietnam: Ninh Binh [Nguyen et al., 2007].

\section{Delta pyriforme pyriforme (Fabricius, 1775)}

Vespa petiolate Fabricius, 1775: 278; Vespa pyriformis Fabricius, 1775: 371; Vespa petiolata Fabricius, 1781: 467; Sphex pyri- 
formis Christ, 1791: 312; Sphex rubicunda Christ, 1791: 316; Sphex thoracica Christ, 1791: 324; Eumenes piriformis Schulz, 1912: 88, 101; Eumenes maxillosus var. petiolatus; Bequaert, 1918 59, 63; Eumenes maxillosus var. pyriformis Bequaert, 1926: 562 564; Eumenes pyriformis pyriformis; Iwata, 1965: 105; Delta pyriformis; Giordani Soika, 1975: 393; Delta pyriforme pyriforme Gusenleitner, 1988: 184.

DISTRIBUTION. India: Arunachal Pradesh, Gujarat, Karnataka, Kerala, Orissa, Rajasthan, Malabar, Sikkim, Tamil Nadu, West Bengal; Bhutan; Cambodia; China; Hawaii; Hong Kong; Indonesia; Malaysia; Moluccas; Myanmar; Nepal; New Guinea; Sri Lanka; Taiwan; Thailand; Vietnam [Srinivasan, Girish, 2010].

NOTE. Srinivasan and Girish [2010] recorded this species from Vietnam, but did not show exact locality.

\section{Ectopioglossa Perkins, 1912}

10. Ectopioglossa keiseri nigra Giordani Soika, 1993

Ectopioglossa keiseri nigra Giordani Soika, 1993: 159.

DISTRIBUTION. Vietnam: Hoa Binh [Giordani Soika, 1993], Quang Ninh.

NOTE. New record for Quang Ninh Province $\left(2 \sigma^{\top} \sigma^{\top} 19\right.$ Hong Thai Tay, Dong Trieu, 208'슬 $106^{\circ} 40^{\prime} \mathrm{E}, \mathrm{h}=188 \mathrm{~m}$, 26.VI.2013, PP).

Eumenes Latreille, 1802

\section{Eumenes flavopictus continentalis} (Zimmermann, 1931)

Eumenes arcuata continentalis Zimmermann, 1931: 203; Eumenes arcuatus (Fabricius) Smith, 1861: 126; Eumenes arcuatus var. flavopictus (Blanch.) Schulthess, 1914: 263; Eumenes flavopictus continentalis van de Vecht, 1972: 36-39.

DISTRIBUTION. India: Sikkim, Assam; Myanmar: Tenasserim; Malaya; Thailand: Siam; China: Canton, Hainan; Indonesia: Sunda Strait, Bangka, Sumatra; Vietnam: ChiemHoa, Phu Quoc, Hanoi [van der Vecht, 1959b].

\section{Eumenes quadratus quadratus F. Smith, 1852}

Eumenes quadratus Smith, 1852: 37; Eumenes reflexus Sickmann, 1894: 230.

DISTRIBUTION. China; Laos; Korea; Vietnam [Bequaert, 1928].

NOTE. Bequaert [1928] recorded this species from Vietnam, but did not show exact locality.

\section{Euodynerus Dalla Torre, 1904}

\section{Euodynerus dantici violaceipennis Giordani Soika, 1973}

Euodynerus dantici violaceipennis Giordani Soika, 1973: 114.

DISTRIBUTION. China: Jianxi (Canton, Soochow); Russian Far East; Korea; Japan: Honshu, Awaji-shima, Kyushu; Vietnam: Cao Bang, Lao Cai, Son La [Dang et al., 2012], Thai Binh.

NOTE. New record for Thai Binh Province $\left(10^{\top} 11_{+}-\right.$ Hung Nhan, Hung Ha, $20^{\circ} 31^{\prime} \mathrm{N} 106^{\circ} 11^{\prime} \mathrm{E}$, h $=2 \mathrm{~m}$, 25.VI.2013, PP).

\section{4 (1). Euodynerus nipanicus nipanicus (von Schulthess, 1908)}

Lionotus tomentosus var. nipanicus Schulthess, 1908: 287 288; Euodynerus notatus Yamane e Tano, 1987: 335-336; Euodynerus notatus ssp. 2, 3; Yamane, 1979: 23-24; Euodynerus notatus nipanicus Yamane e Tano, 1987: 337-338; Euodynerus nipanicus Giordani Soika, 1986:135-138.
DISTRIBUTION. China; Korea; Japan; Thailand; Vietnam: Dien Bien [Dang et al., 2012], Nam Dinh, Quang Ninh, Thai Binh

NOTE. New records for the Provinces Nam Dinh $\left(10^{7}-\right.$ Xuan Thuy NP, Xuan Thuy, 20 $0^{\circ} 13^{\prime} \mathrm{N} 106^{\circ} 31^{\prime} \mathrm{E}, 22 . V I .2013$, PP), Quang Ninh ( $10^{\top} 1$ 우 - Phuong Dong, Uong Bi, $21^{\circ} 04^{\prime} \mathrm{N}$ $106^{\circ} 45^{\prime} \mathrm{E}, \mathrm{h}=123 \mathrm{~m}, 26$.VI.2013, PP) and Thai Binh (2우 Hung Nhan, Hung Ha, $20^{\circ} 31^{\prime} \mathrm{N}, 106^{\circ} 11^{\prime} \mathrm{E}, \mathrm{h}=2 \mathrm{~m}$, 25.VI.2013, PP; $19-$ Dong Minh, Tien Hai, 20³9’ N $106^{\circ} 58^{\prime}$ E, 21.VI.2013, PP; $30^{\top} \sigma^{\top}$ - Phuong Cong, Tien Hai, $20^{\circ} 38^{\prime} \mathrm{N} 106^{\circ} 56^{\prime} \mathrm{E}, 21 . \mathrm{VI} .2013$, PP).

\section{4 (2). Euodynerus nipanicus tonkinensis Giordani Soika, 1973}

Odynerus (Pareuodynerus) notatus ssp. tonkinensis Giordani Soika, 1973: 118; Euodynerus nipanicus tonkinensis Giordani Soika, 1994: 250-251.

DISTRIBUTION. China: Kunming, Shanghai, Canton; Vietnam: Hoa Binh, Lao Cai [Giordani Soika, 1973, 1994; Dang et al., 2012], Hanoi.

NOTE. New record for Hanoi ( 3 우 — Nghia Do, Cau Giay, $21^{\circ} 03^{\prime} \mathrm{N} 105^{\circ} 50^{\prime} \mathrm{E}, \mathrm{h}=9 \mathrm{~m}, 29$.VI.2013, PP).

\section{Euodynerus trilobus (Fabricius, 1787)}

Vespa triloba Fabricius, 1787: 290; Odynerus trilobus Lepeietier de Saint Fargeau, 1841: 635; Pseudepipona triloba Iwata, 1939: 62; Euodynerus trilobus van der Vecht et Fischer, 1972: 101.

DISTRIBUTION. British Indian Ocean Territories; Mauritius; Réunion; China; Malaysia: Peninsular Malaysia; Taiwan; Japan: Ryukyu Is.; Indonesia: Java; Vietnam: Bac Giang, Hanoi, Ha Tinh, Hoa Binh, Phu Tho, Vinh Phuc [Giordani Soika, 1994; Dang, Nguyen, Kojima, 2012], Nam Dinh, Thai Binh.

NOTE. New records for the Provinces Nam Dinh (1 $9-$ Xuan Thuy NP, Xuan Thuy, $20^{\circ} 13^{\prime} \mathrm{N} 106^{\circ} 31^{\prime} \mathrm{E}$, 21.VI.2013, PP) and Thai Binh (1은 Hong Minh, Hung Ha, $20^{\circ} 35^{\prime} \mathrm{N}$ $106^{\circ} 08^{\prime} \mathrm{E}, \mathrm{h}=3 \mathrm{~m}, 23$.VI.2013, PP; $10^{\top}$ - Hung Nhan, Hung Ha, $20^{\circ} 31^{\prime} \mathrm{N} 106^{\circ} 11^{\prime} \mathrm{E}, \mathrm{h}=2 \mathrm{~m}, 25$.VI.2013, PP).

Lissodynerus Giordani Soika, 1993

16. Lissodynerus septemfasciatus feanus (Giordani Soika, 1941)

Ancistrocerus (Ancistrocerus) septemfasciatus var. feanus Giordani Soika, 1941: 239; Lissodynerus septemfasciatus feanus Giordani Soika, 1973: 119.

DISTRIBUTION. Malaysia: Malaya Penninsular; Vietnam: Hoa Binh [Giordani Soika, 1941, 1973, 1994].

Malayepipona Gordani Soika, 1993

17. Malayepipona clypeata

Nguyen et Carpenter, 2013

Malayepipona clypeata Nguyen et Carpenter, 2013: 729-731. DISTRIBUTION. Vietnam: Bac Can [Nguyen, Carpenter, 2013].

18. Malayepipona furva

Nguyen et Carpenter, 2013

Malayepipona furva Nguyen et Carpenter, 2013: 732-733.

DISTRIBUTION. Vietnam: Tam Dao [Nguyen, Carpenter, 2013].

19. Malayepipona malickyi (Gusenleitner, 2010)

Indodynerus malickyi Gusenleitner, 2010: 696-698; Malayepipona assamensis manipurensis (Gordani Soika) Gusenleitner, 2012: 1047.

DISTRIBUTION. Vietnam: Tam Dao [Gusenleitner, 2010; Nguyen, Carpenter, 2013]. 


\section{Malayepipona seomyty}

Nguyen et Carpenter, 2013

Malayepipona seomyty Nguyen et Carpenter, 2013: 731-732. DISTRIBUTION. Vietnam: Lao Cai [Nguyen, Carpenter, 2013].

Nortozumia van der Vecht, 1937

21. Nortozumia picea van der Vecht, 1963

Nortozumia picea van der Vecht, 1963: 40. 1963].

DISTRIBUTION. Vietnam: Phu Quoc [van der Vecht,

Orancistrocerus van der Vecht, 1963

22. Orancistrocerus aterrimus nigriceps van der Vecht, 1963

Orancistrocerus aterrimus nigriceps van der Vecht, 1963: 102, 106. 1963].

DISTRIBUTION. Vietnam: Phu Quoc [van der Vecht,

Parancistrocerus Bequaert, 1925

23. Parancistrocerus androcles

(Meade Waldo, 1910)

Odynerus androcles Meade Waldo, 1910: 103; Ancistrocerus (Ancistrocerus) androcles Giordani Soika, 1941: 236; Ancistrocerus horni Sonan, 1938: 262; Parancistrocerus androcles Gusenleitner, 1988: 178.

DISTRIBUTION. India; Sri Lanka: Ceylon; Myanmar; Thailand; Malaysia: Peninsular Malaysia, Sabah; Singapore; Philippines; Indonesia: Sumatra, Java, Sulawesi; Vietnam [Nugroho et al., 2011].

NOTE. Nugroho with the coauthors [2011] recorded this species from Vietnam, but did not show exact locality.

\section{Parancistrocerus assamensis (Meade-Waldo, 1910)}

Odynerus assamensis Meade-Waldo, 1910: 103; Parancistrocerus assamensis Giordani Soika, 1994: 155, 171.

DISTRIBUTION. India; Nepal; Myanmar; Laos; Vietnam [Giordani Soika, 1994].

NOTE. Giordani Soika [1994] recorded this species from Vietnam, but did not show exact locality. He stated the distribution of this species as follows "Viet Nam: loc. indecifrabile, 1Q (coll.-AGS)".

25. Parancistrocerus insolitus Gusenleitner, 2012

Parancistrocerus insolitus Gusenleitner, 2012: 1045-1046. 2012].

DISTRIBUTION. Vietnam: Cao Bang [Gusenleitner,

\section{Pareumenes (de Saussure, 1855)}

26. Pareumenes quadrispinosus (de Saussure, 1855)

Eumenes quadrispinosus de Saussure, 1855: 134; Eumenes quadrispinosa Bingham, 1897: 336; Pareumenes quadrispinosa de Saussure, 1904: 19.

DISTRIBUTION. India: Arunachal Pradesh, Assam, Karnataka, Sikkim, Tamil Nadu, Uttarakhand, West Bengal; Bhutan; China; Hong Kong; Indonesia; Malaysia; Myanmar; Vietnam: Hoa Binh, Quang Ninh [Srinivasan, Girish, 2010].

NOTE. Srinivasan and Girish [2010] recorded this species from Vietnam, but did not show exact locality. The present list does not therefore arrange distributional localities of the species as new distributional records $\left(10^{7}-\mathrm{Hoa}\right.$ Binh, Mai Chau, $20^{\circ} 39^{\prime} \mathrm{N} 105^{\circ} 04^{\prime} \mathrm{E}, \mathrm{h}=287 \mathrm{~m}, 3$.VI.2012, PP; 1 으 Quang Ninh, Yen Tu NP, $21^{\circ} 02^{\prime} \mathrm{N} 106^{\circ} 36^{\prime} \mathrm{E}, \mathrm{h}=$
257 m, 27.VI.2013, PP).

Phimenes Giordani Soika, 1992

27. Phimenes flavopictus continentalis (Zimmermann, 1931)

Eumenes arcuata continentalis Zimmermann, 1931: 205, 224, 226; Eumenes flavopictus continentalis van der Vecht, 1959: 36; Phimenes flavopictum continentale Gusenleitner, 2006: 694.

DISTRIBUTION. India; Nepal; China; Myanmar; Thailand; Malaysia: Peninsular Malaysia; Indonesia: Sumatra, Krakatau; Vietnam: Hoa Binh, Quang Ninh [Nugroho et al., 2011$]$.

NOTE. This species was recorded from Vietnam, but without exact locality [Nugroho et al., 2011]. The present list does not thus affirm distributional localities of the species as new distributional records $(1+$ - Hoa Binh, Mai Chau, $20^{\circ} 39^{\prime} \mathrm{N} 105^{\circ} 04^{\prime} \mathrm{E}, \mathrm{h}=287 \mathrm{~m}, 3$.VI.2012, PP; $1 \mathrm{O}^{\top}$ - Quang Ninh, Hong Thai Tay, Dong Trieu, $20^{\circ} 89^{\prime} \mathrm{N} 106^{\circ} 40^{\prime} \mathrm{E}, \mathrm{h}=$ 188 m, 26.VI.2013, PP).

\section{Pseudozumia de Saussure, 1875}

28. Pseudozumia indica indica (de Saussure, 1855)

Montezumia indica de Saussure, 1855: 167; Pseudozumia indica: Giordani Soika, 1941: 164, 166.

DISTRIBUTION. India; Sri Lanka; China; Taiwan; Malaysia; Vietnam; Indonesia: Java, Lombok, Bali; Vietnam: Hoa Binh [Giordani Soika, 1941].

Pseumenes Giordani Soika, 1935

29. Pseumenes depressus depressus (de Saussure, 1855)

Eumenes depressus de Saussure, 1855: 135; Pareumenes depressa; Dalla Torre, 1904: 19; ?Pareumenes quadrispinosa var. depressa Dover, 1931: 252; Pareumenes "quadrispinosus Saussure" Piel, 1935: 115; Pseumenes depressus depressus van der Vecht, 1963: 25-26.

DISTRIBUTION. India; Myanmar: Tenasserim; China; Hong Kong; Indonesia: Siam; Malaysia: Sarawak; Vietnam: Hanoi [van der Vecht, 1963].

\section{Rhynchium Spinola, 1806}

\section{Rhynchium brunneum (Fabricius, 1793)}

Vespa brunnea Fabricius, 1793: 264; Rynchium khandalense Dusmet, 1930: 105; Rhynchium brunneu: Bingham, 1897: 355-356.

DISTRIBUTION. India: Arunachal Pradesh, Delhi, Maharashtra, Meghalaya, Sikkim, Uttarakhand, Tamil Nadu, West Bengal; Afghanistan; Bangladesh; Nepal, Pakistan; Sri Lanka; Iran; Myanmar; Malaysia; Thailand; Cambodia; Society Is.; Marquesas Is.; Indonesia: Tanimbar Islands; Vietnam: Hoa Binh, Quang Ninh, Thai Binh [Nugroho et al., 2011].

NOTE. This species was recorded from Vietnam, but without exact locality [Nugroho et al., 2011]. So the present list gives the distributional record of the species in two provinces in Vietnam as stated above, but does not assert distributional localities of the species as new distributional records $\left(2 \sigma^{\top} \sigma^{\top}\right.$ - Thai Binh, Hung Nhan, Hung Ha, $20^{\circ} 31^{\prime} \mathrm{N}$ $106^{\circ} 11^{\prime} \mathrm{E}, \mathrm{h}=2 \mathrm{~m}, 25$.VI.2013; $1 \mathrm{\sigma}^{\mathrm{T}}$ - Thai Binh, Hong Minh, Hung Ha, $20^{\circ} 35^{\prime} \mathrm{N} 106^{\circ} 08^{\prime} \mathrm{E}, \mathrm{h}=3 \mathrm{~m}, 23-24$. VI.2013; $1{ }^{\circ}$ — Thai Binh, Dong Minh, Tien Hai, 20³9’ N $106^{\circ} 58^{\prime} \mathrm{E}$, 21.VI.2013, PP; $2 \sigma^{7} \sigma^{7} 1$ — Q Quang Ninh, Hong Thai Tay, Dong Trieu, $20^{\circ} 89^{\prime} \mathrm{N} 106^{\circ} 40^{\prime} \mathrm{E}, \mathrm{h}=188 \mathrm{~m}, 26$.VI.2013, PP; $10^{7} 1$ ㄴ Hoa Binh, Mai Chau, $20^{\circ} 39^{\prime} \mathrm{N} 105^{\circ} 04^{\prime} \mathrm{E}, \mathrm{h}=287$ m, 3.VI.2012, PP). 


\section{Rhynchium haemorrhoidale (Fabricius, 1775)}

Vespa haemorrhoidalis Fabricius, 1775: 366; Odynerus dimidiatus Guérin-Méneville, 1834: 503; Rygchium haemorrhoidale: de Saussure, 1852: 109; Rygchium parentissimum de Saussure, 1852: 111; Rhynchium haemorrhoidale van der Vecht, 1963: 11 .

DISTRIBUTION. Myanmar; Thailand; Cambodia; Malaysia: Peninsular Malaysia, Sarawak, Sabah; Singapore; Indonesia: Sumatra, Java, Bali, Sumbawa, Papua; Vietnam: Hoa Binh, Quang Ninh [Nugroho et al., 2011].

NOTE. Like $R$. brunneum, this species also recorded from Vietnam,but without exact locality [Nugroho et al., 2011]. The distributional localities of the species are thus listed here not affirmed as newly distributional records $(1+$ Quang Ninh, Hong Thai Tay, Dong Trieu, $20^{\circ} 89^{\prime} \mathrm{N} 106^{\circ} 40^{\prime} \mathrm{E}$, $\mathrm{h}=188 \mathrm{~m}, 26$. VI.2013, PP; $10^{7}$ - Hoa Binh, Mai Chau, $20^{\circ} 39^{\prime} \mathrm{N} 105^{\circ} 04^{\prime} \mathrm{E}, \mathrm{h}=287 \mathrm{~m}, 3$.VI.2012, PP).

\section{Subancistrocerus de Saussure, 1855}

\section{Subancistrocerus sichelii (de Saussure, 1855)}

Odynerus sichelii de Saussure, 1855: 206; Odynerus intendens Walker, 1860: 304; Ancistrocerus sichelii Giordani Soika, 1941: 242; Subancistrocerus sichelii Iwata, 1965: 105; Subancistrocerus tristis Giordani Soika, 1991: 41, 50; Subancistrocerus sichelii: Giordani Soika, 1994: 13, 33-35.

DISTRIBUTION. Mauritius; Seychelles; India; Sri-Lanka; Chagos Archipelago; Nepal; Myanmar; Thailand; Cambodia; Malaysia; Singapore; Vietnam: Pleiku [Giordani Soika, 1994].

\section{Zethus Fabricius, 1804}

33. Zethus nigerrimus Gusenleitner, 2001

Zethus nigerrimus Gusenleitner, 2001: 659-661.

DISTRIBUTION. Malaysia; Vietnam: Vinh Phuc [Gusenleitner, 2001].

\section{Subfamily Stenogastrinae}

Cochlischnogaster Dong et Otsuka, 1997

\section{Cochlischnogaster daduganensis}

Dong et Otsuka, 1997 210

Cochlischnogaster dadugangensis Dong et Otsuka, 1997: 205,

DISTRIBUTION. India: Arunachal Pradesh; China: Yunnan; Vietnam: Phu Tho [Nguyen, 2007].

\section{Cochlischnogaster spatulata}

(Carpenter et Starr, 2000)

Chalogaster spatulata Carpenter et Starr, 2000: 1-3; Cochlischnogaster spatulata Carpenter, 2001: 28.

DISTRIBUTION. Thailand: Chieng Mai, Loei; Vietnam: Quang Binh [Carpenter, Starr, 2000; Nguyen et al., 2005; Nguyen, 2007].

Eustenogaster van der Vecht, 1969

\section{Eustenogaster hauxwellii (Bingham, 1894)}

Ischnogaster rufo-maculata Bingham, 1894: 385; Ischnogaster hauxwellii Bingham, 1894: 386; Ischnogaster hauxwelli von Dalla Torre, 1904:84; ?Stenogaster bicarinata Dover et Rao, 1922: 242; Eustenogaster hauxwellii Iwata, 1976: 274.

DISTRIBUTION. India: Sikkim; Myanmar; Thailand; Malaysia: Sarawak; Singapore; Indonesia: Java, Sumatra, Bangka, Kalimantan; Philippines: Mindanao; Vietnam: Dak Lak, Dong Nai, Lam Dong, Quang Nam, Tay Ninh [Nguyen et al., 2005; Nguyen, 2007].
4. Eustenogaster nigra Saito et Nguyen, 2006

Eustenogaster nigra Saito et Nguyen, 2006: 4, 6.

DISTRIBUTION. China: Yunan; Vietnam: Hanoi, Ha Tinh, Hai Phong, Hoa Binh, Lang Son, Ninh Binh, Nghe An, Phu Tho, Quang Ninh (new record), Thai Nguyen, Vinh Phuc [Nguyen, Khuat, 2004; Saito et al., 2006; Nguyen, 2007; Nguyen, Pham, 2011].

Saito et al. [2006] stated that: "Nguyen and Khuat [2004] published the name in quotation marks, ..., and they gave its diagnostic characters in a key, ... There was no intention to make the name available, as it is not under the current International Code of Zoological Nomenclature [ICZN, 1999] That is, Nguyen and Khuat [2004] neither made an explicit statement that the name is new [ICZN, 1999: Article 16] nor fixed the name-bearing type [ICZN, 1999: Article 16.4]". So, they validated the name of the species, and described the species as a new species to Vietanm with the details of both female and male together with the nest. The species has been only recorded from the northern part of the country (in 10 provinces). Outside Viet Nam, this species has also been recorded from Southern China. In present list, we add a new distributional locality as stated above (6우 - Hai Phong, Cat Ba NP, Cat Hai, $20^{\circ}$ $45^{\prime} \mathrm{N} 107^{\circ} 05^{\prime} \mathrm{E}, \mathrm{h}=210 \mathrm{~m}, 17-18 . \mathrm{III} .2012$, PP; 1 우 Quang Ninh, Phuong Dong, Uong Bi, $21^{\circ} 04^{\prime} \mathrm{N} 106^{\circ} 45^{\prime} \mathrm{E}, \mathrm{h}$ $=188 \mathrm{~m}$, 26.VI.2013, PP).

\section{Eustenogaster scitula (Bingham, 1894)}

Ischnogaster scitula Bingham, 1897: 377, 379; Stenogaster scitula var. assamensis Dover et Rao, 1922: 240; Stenogaster seitula Lee, 1982: 130; Eustenogaster scitula Das et Gupta, 1984(1983): 402

DISTRIBUTION. India: Assam, Meghalaya, Sikkim; Myanmar; Malaysia; Vietnam: Lam Dong, Quang Tri, Quang Binh, Quang Nam, Thua Thien Hue [Nguyen et al., 2005; Nguyen, 2007].

\section{Eustenogaster vietnamensis Saito, 2009}

Eustenogaster vietnamensis Saito, 2009: 19.

DISTRIBUTION. Vietnam: Vinh Phuc [Saito, 2009].

Liostenogaster van der Vecht, 1969

7. Liostenogaster filicis Turillazzi, 1999

Liostenogaster filicis Turillazzi, 1999: 343-344.

DISTRIBUTION. Malaysia: Malaysian Peninsula; Myanmar: Tenasserim; Laos: Vientiane; Vietnam: Hanoi, Ha Giang, Ha Tinh, Lai Chau, Lam Dong, Quang Tri, Quang Nam, Son La, Tay Ninh, Thua Thien Hue [Nguyen, 2007; Nguyen, Pham, 2011].

\section{Liostenogaster nitidipennis (de Saussure, 1853)}

Ischnogaster nitidipennis de Saussure, 1853: 10; Parischnogaster nitidipennis von Schulthess, 1927: 88; Stenogaster nitidipennis Dover, 1929: 46; Liostenogaster nitidipennis Iwata, 1976: 275.

DISTRIBUTION. Myanmar; Thailand; Malaysia: Peninsular Malaysia, Sabah; Singapore; Indonesia: Java, Kalimantan, Sumatra; Philippines: Luzon, Palawan; Vietnam: Quang Nam [Nguyen, 2007].

Parischnogaster von Schulthess, 1914

9. Parischnogaster mellyi (de Saussure, 1852)

Ischnogaster mellyi de Saussure, 1852: 25; Ischnogaster nigrifrons Smith, 1857: 113; Stenogaster nigrifrons; Dover et Rao, 1922: 241; Parischnogaster mellyi von Schulthess, 1927: 82, 87; Parischnogaster nigrifrons von Schulthess, 1927: 83, 87; - Dong et Otsuka, 1997: 205; Stenogaster mellyi Pagden, 1962: 95. 
DISTRIBUTION. India: Assam, Meghalaya, Sikkim; China: Yunnan; Myanmar; Thailand; Malaysia: Peninsular Malaysia, Sabah, Sarawak; Singapore; Indonesia: Sumatra, Java, Kalimantan, Bangka; Philippines: Mindanao; Vietnam: Can Tho, Dong Nai, Hanoi, Hoa Binh, Kien Giang, Lai Chau, Lam Dong, Nghe An, Ninh Thuan, Quang Tri, Son La, Tay Ninh, Vinh Phuc [Carpenter, Kojima, 1997a; Nguyen et al., 2005; Nguyen, 2007; Nguyen, Pham, 2011].

10. Parischnogaster striatula (du Buysson, 1905)

Ischnogaster striatulus du Buysson, 1905: 282; ?Ischnogaster clypealis Cameron, 1906: 122; Ischnogaster foveatus du Buysson, 1907: 80; ?Ischnogaster levifoveatus Meade-Waldo, 1914: 462; ?Parischnogaster costulatus von Schulthess, 1927: 82.

DISTRIBUTION. Thailand; Malaysia: Peninsular Malaysia, Sarawak; Indonesia: Sumatra, Kalimantan, Salawaty, Java; Vietnam: Lam Dong, Quang Nam [Nguyen, 2007].

Subfamily Polistinae

Polistes Latreille, 1802

1. Polistes affinis Gusenleitner, 2006

Polistes affinis Gusenleitner, 2006: 671-672.

DISTRIBUTION. Laos: Phongsaly; Vietnam: Lao Cai, Vinh Phuc [Nguyen, 2007; Nguyen et al., 2011].

2. Polistes brunetus Nguyen et Kojima, 2013

Polistes brunetus Nguyen et Kojima, 2013: 57, 59;

DISTRIBUTION. Vietnam: Bac Can, Bac Giang, Ha

Giang, Ha Tinh, Hoa Binh, Lang Son, Ninh Binh, Nghe An, Phu Tho, Son La, Thanh Hoa, Vinh Phuc [Nguyen, Kojima, 2013]

3. Polistes clandestinus

Nguyen, Kojima et Saito, 2011

Polistes (Polistella) clandestinus Nguyen, Kojima et Saito, 2011: 19-21. $2011]$.

DISTRIBUTION. Vietnam: Lao Cai [Nguyen et al.,

\section{Polistes curcipunctum}

Nguyen, Kojima et Saito, 2011

Polistes (Polistella) curcipunctum Nguyen, Kojima et Saito, 2011: 15-17.

DISTRIBUTION. Vietnam: Ha Tinh, Quang Nam, Vinh Phuc [Nguyen et al., 2011].

\section{Polistes dawnae Dover et Rao, 1922}

Polistes dawnae Dover et Rao, 1922: 248.

DISTRIBUTION. Northern Myanmar; Vietnam: Dien Bien, Hoa Binh, Lai Chau [Nguyen, 2007; Nguyen, Pham, 2011; Nguyen et al., 2011].

6. Polistes delhiensis Das et Gupta, 1984

Polistes delhiensis Das et Gupta, 1984: 410.

DISTRIBUTION. India: Delhi; Vietnam: Ha Giang Hoa Binh, Son La, Phu Tho, Vinh Phuc [Nguyen, 2007; Nguyen et al., 2011].

7. Polistes gigas (Kirby, 1826) 469

Cyclostoma gigas Kirby, 1826: 36; Polistes gigas Sonan, 1942:

DISTRIBUTION. India: Sikkim; Northern China; Taiwan, Vietnam: Hanoi, Hai Phong, Ha Tinh, Hoa Binh, Lang Son, Ninh Binh, Nghe An, Phu Tho, Quang Ninh, Hoa, Quang Nam, Vinh Phuc [Nguyen et al., 2005; Nguyen, 2007; Nguyen, Pham, 2011].
8. Polistes gilvus Nguyen, Kojima et Saito, 2011

Polistes (Polistella) gilvus Nguyen, Kojima et Saito, 2011: 17-19.

DISTRIBUTION. Vietnam: Lao Cai, Son La [Nguyen et al., 2011]

9. Polistes horrendus Gusenleitner, 2006

Polistes horrendus Gusenleitner, 2006: 671.

DISTRIBUTION. Laos: Phongsaly; Vietnam: Hoa Binh [Nguyen, 2007; Nguyen et al., 2011; Nguyen, Pham, 2011].

10. Polistes japonicus de Saussure, 1858

Polistes japonicus de Saussure, 1858: 260.

DISTRIBUTION. China; Korea; Japan; Vietnam: Ha Giang, Hanoi, Ha Tinh, Hai Phong, Hoa Binh, Lai Chau, Ninh Binh, Nghe An, Phu Tho, Quang Tri, Quang Nam, Son La, Thanh Hoa, Thua Thien Hue, Vinh Phuc [Nguyen et al., 2005; Nguyen, 2007; Nguyen, Pham, 2011], Quang Ninh.

NOTE. New record for Quang Ninh Province (19 Hong Thai Tay, Dong Trieu, $20^{\circ} 89^{\prime} \mathrm{N} 106^{\circ} 40^{\prime} \mathrm{E}, \mathrm{h}=188 \mathrm{~m}$, 26.VI.2013, PP; $1{ }^{\circ}$ - Yen Tu NP, Uong Bi, $21^{\circ} 02^{\prime} \mathrm{N}$ $106^{\circ} 36^{\prime} \mathrm{E}, \mathrm{h}=257 \mathrm{~m}, 27 . \mathrm{VI} .2013$, PP).

11. Polistes khasianus Cameron, 1900

Polistes khasianus Cameron, 1900: 413.

DISTRIBUTION. India: Haryana, Meghalaya, West Bengal; Vietnam: Ha Tinh, Hoa Binh, Son La [Nguyen, 2007; Nguyen, Pham, 2011].

\section{Polistes lepcha Cameron, 1900}

Polistes khasianus Cameron, 1900: 415; Polistes lepcha Cameron, 1900: 506.

DISTRIBUTION. India: Haryana, Meghalaya, Manipur; Vietnam: Lai Chau, Lao Cai [Nguyen, 2007; Nguyen et al., $2011]$.

13. Polistes mandarinus de Saussure, 1853

Polistes (Polistella) mandarinus de Saussure, 1853: 58.

DISTRIBUTION. North China; Korea; Vietnam: Ha Giang, Phu Tho, Thua Thien Hue, Vinh Phuc [Nguyen, 2007; Nguyen et al., 2011].

\section{Polistes nigerrimus Gusenleitner, 2006}

Polistes nigerrimus Gusenleitner, 2006: 674.

DISTRIBUTION. Vietnam: Thanh Hoa, Vinh Phuc [Carpenter, 1996; Nguyen et al., 2011].

\section{Polistes nigritarsis Cameron, 1900}

Polistes nigritarsis Cameron, 1900: 413.

DISTRIBUTION. India: West Bengal, Meghalaya, Kerala, Karnataka, Tamil Nadu; Vietnam: Lai Chau, Lam Dong, Son La, Thua Thien Hue [Nguyen, 2007; Nguyen, Pham, 2011].

\section{Polistes nipponensis Pérez, 1905}

Polistes nipponensis Pérez, 1905: 82; Polistes yamanakai Sonan, 1937:169.

DISTRIBUTION. China; Japan: Kanagawa; Vietnam: Hai Phong, Hoa Binh, Ninh Binh, Vinh Phuc [Nguyen, 2007; Nguyen et al., 2011].

\section{Polistes olivaceus (De Geer, 1773)}

Vespa olivacea De Geer, 1773: 582; Polistes olivaceus de Saussure, 1853: 89 .

DISTRIBUTION. Madagascar; Reunion; Mauritius; Tanzania; Amirantes; Seychelles; Chagos Archipelago; Egypt; 
Oman; Iran; Afghanistan; India; Sri Lanka; Nepal; Myanmar; southern China; Japan; Philippines; Laos; Cambodia; Thailand; Malaysia; Singapore; Marianas; New Caledonia; Fiji; Tongas; Samoa; Society Is.; Tuamotu Archipelago; Marquesas; Chile: Easter Is.; U.S.A.: Hawaii; Australia; New Zealand; Indonesia: Kalimantan. Vietnam: Dien Bien, Ha Noi, Hoa Binh, Kien Giang, Lao Cai, Nghe An, Quang Binh, Son La, Thua Thien Hue, Vinh Phuc, Yen Bai [Carpenter, 1996; Nguyen et al., 2005; Nguyen, 2007], Quang Ninh, Thai Binh. NOTE. New records for the Provinces Quang Ninh $\left(2\right.$ + 0 - Hong Thai Tay, Dong Trieu, $20^{\circ} 89^{\prime} \mathrm{N} 106^{\circ} 40^{\prime} \mathrm{E}, \mathrm{h}=$ $188 \mathrm{~m}, \mathrm{PP} ; 3$ 우 — Phuong Dong, Uong Bi, $21^{\circ} 04^{\prime} \mathrm{N} 106^{\circ} 45^{\prime} \mathrm{E}$, $\mathrm{h}=123 \mathrm{~m}, 26$. VI.2013, PP; 2 우 - Yen Tu NP, Uong Bi, $\left.21^{\circ} 02^{\prime} \mathrm{N} 106^{\circ} 36^{\prime} \mathrm{E}, \mathrm{h}=257 \mathrm{~m}, 27 . \mathrm{VI} .2013, \mathrm{PP}\right)$ and Thai Binh $\left(10^{\top} 17\right.$ 우 - Hong Minh, Hung Ha, $20^{\circ} 35^{\prime} \mathrm{N} 106^{\circ} 08^{\prime} \mathrm{E}$, $\mathrm{h}=3 \mathrm{~m}, 23-24 . V I .2013$, PP, nest No201303; 21우 — Dong Minh, Tien Hai, $20^{\circ} 39^{\prime} \mathrm{N} 106^{\circ} 58^{\prime} \mathrm{E}, 21 . \mathrm{VI} .2013$, PP, nest No 201301-201302; 6우 — Hung Nhan, Hung Ha, 2031ㅅN $106^{\circ} 11^{\prime} \mathrm{E}, \mathrm{h}=2 \mathrm{~m}, 25 . \mathrm{VI} .2013$, PP).

\section{Polistes paco Nguyen, Kojima et Saito, 2011}

Polistes (Polistella) paco Nguyen, Kojima et Saito, 2011: 14-15. 2011].

DISTRIBUTION. Vietnam: Hoa Binh [Nguyen et al.,

19. Polistes reliciniclypeus

Nguyen, Kojima et Saito, 2011

Polistes (Polistella) reliciniclypeus Nguyen, Kojima et Saito, 2011: 9-14.

DISTRIBUTION. Vietnam: Dien Bien, Hoa Binh, Lai Chau, Lao Cai [Nguyen et al., 2011].

20. Polistes rothneyi quatei van der Vecht, 1968

Polistes rothneyi quatei van der Vecht, 1968: 106

DISTRIBUTION. Pakistan; India: Jammu and Kashmir, Himachal Pradesh, Uttar Pradesh; Nepal; Myanmar; Indonesia: Sumatra; China; Taiwan; Japan; Korea; Vietnam: Cao Bang, Hoa Binh, Kontum, Lang Son, Ninh Binh, Nghe An, Phu Tho, Vinh Phuc [van der Vecht, 1968; Carpenter, 1996; Nguyen et al., 2005; Nguyen, 2007; Nguyen, Pham, 2011].

\section{Polistes sagittarius de Saussure, 1853}

Polistes sagittarius de Saussure, 1853b: 56; Polistes sagittarius var (or subsp.) indonesicus Bequaert, 1940: 267; Polistes strigosus baliensis Gusenleitner, 2000: 939.

DISTRIBUTION. India: Himachal Pradesh, Delhi, Kerala, Uttaranchal, Sikkim, West Bengal, Assam, Nagaland, Manipur, Tripura; Nepal; China; Hong Kong; Myanmar; Thailand; Malaysia; Singapore; Philippines: Palawan; Indonesia: Sumatra, Kalimantan, Java, Bali, Lombok, Flores, Sumbawa, Sumba, Sulawesi; Vietnam: Binh Duong, Dien Bien, Hanoi, Ha Giang, Hoa Binh, Lai Chau, Nghe An, Son La, Vinh Phuc [Nguyen et al., 2005; Nguyen, 2007; Nguyen, Pham, 2011].

\section{Polistes santoshae Das et Gupta, 1989}

Polistes santoshae Das et Gupta, 1989: 72.

DISTRIBUTION. India: Sikkim, Meghalaya; Nepal; Vietnam: Quang Nam [Nguyen, 2007; Nguyen et al., 2011].

23. Polistes stigma (Fabricius, 1793)

Vespa stigma Fabricius, 1793: 275; Polistes stigma de Saussure, 1853: 64

DISTRIBUTION. Thailand; Malaysia; Singapore; Taiwan; Indonesia: Sumatra, Java, Bali, Lombok; Vietnam: Hanoi, Hai Phong, Hoa Binh, Nghe An, Ninh Binh, Thanh
Hoa, Vinh Phuc [Nguyen et al., 2005; Nguyen, 2007], Quang Ninh.

NOTE. New record for Quang Ninh Province (11우 $20^{\top} \sigma^{\top}-$ Yen Tu NP, Uong Bi, $21^{\circ} 02^{\prime} \mathrm{N} 106^{\circ} 36^{\prime} \mathrm{E}, \mathrm{h}=257$ $\mathrm{m}, 26$.VI.2013, PP, nest No 201304; 1 우 - Phuong Dong, Ung Bi, $21^{\circ} 04^{\prime} \mathrm{N} 106^{\circ} 45^{\prime} \mathrm{E}, \mathrm{h}=123 \mathrm{~m}, 26 . \mathrm{VI} .2013$, PP).

\section{Polistes strigosus Bequaert, 1940}

Polistes strigosus Bequaert, 1940: 269-272.

DISTRIBUTION. China; Taiwan; Vietnam: Ha Giang, Hai Phong, Hanoi, Ha Tinh, Hoa Binh, Nghe An, Quang Tri, Thua Thien Hue, Vinh Phuc [Nguyen, 2007; Nguyen, Pham, 2011].

25. Polistes tenebricosus

Lepeletier de Sait Fargeau, 1836

Polistes tenebricosa Lepeletier de Sait Fargeau, 1836: 529; Polistes sulcatus F. Smith, 1852: 38; Polistes hoplitus de Saussure, 1853b: 55; Polistes rugifrons Cameron, 1900: 412; Polistes varicornis Cameron, 1905b: 67; Polistes javanicus Cameron, 1905b: 68; Polistes tenebricosus var. leopoldi Bequaert, 1934: 9; Polistes tenebricosus var. (or subsp.) nigrosericans Bequaert, 1940: 266.

DISTRIBUTION. India: Jammu and Kashmir, Uttaranchal, Sikkim, West Bengal, Assam, Meghalaya; Nepal; China; Taiwan; Myanmar; Philippines: Luzon, Negros, Sibuyan; Indonesia: Sumatra, Java, Bali, Lombok, Flores, Sumba, Kalimantan, Sulawesi; Vietnam: Bac Can, Ha Tinh, Hoa Binh, Ninh Thuan, Nghe An, Phu Tho, Quang Nam, Vinh Phuc [Carpenter, 1996; Nguyen et al., 2005; Nguyen, 2007], Quang Ninh.

NOTE. New record for Quang Ninh Province (1900 Yen Tu NP, Uong Bi, $21^{\circ} 02^{\prime} \mathrm{N} 106^{\circ} 36^{\prime} \mathrm{E}, \mathrm{h}=257 \mathrm{~m}, 27$.VI. 2013, PP, nest No 201305).

Ropalidia Guérin-Méneville, 1831

26. Ropalidia artifex (de Saussure, 1854)

Icaria artifex de Saussure, 1854: 236; Ropalidia artifex artifex van der Vecht, 1941: 110, 134; Ropalidia artifex var. fuscata van der Vecht, 1941: 136

DISTRIBUTION. Myanmar; Malaysia: Malay Peninsula, Sarawak; Indonesia: Java; Vietnam: Hoa Binh, Lam Dong, Lai Chau, Phu Tho, Quang Tri, Quang Nam, Vinh Phuc [Nguyen et al., 2006b; Nguyen, 2007; Nguyen, Pham, 2011].

\section{Ropalidia bicolorata van der Vecht, 1962}

Paraicaria bicolor Gribodo, 1892 (1891): 249; Ropalidia bicolorata bicolorata van der Vecht, 1962: 38-39; Ropalidia bicolorata parvula van der Vecht, 1962: 38-39; Ropalidia bicolorata shiva Das et Gupta, 1989: 153-154.

DISTRIBUTION. India: Assam, Manipur, Tripura; Myanmar; Thailand; China: Yunnan; Malaysia: Sarawak; Vietnam: Binh Thuan, Dong Nai, Ha Giang, Ha Tinh, Hoa Binh, Lai Chau, Lam Dong, Ninh Thuan, Phu Tho, Quang Binh, Quang Nam, Quang Tri, Son La, Thua Thien Hue [Nguyen et al., 2005, 2006b; Nguyen, 2007; Nguyen, Pham, 2011].

28. Ropalidia birmanica van der Vecht, 1962

Ropalidia taiwana birmanica van der Vecht, 1962: 23.

DISTRIBUTION. Myanmar; Vietnam: Hoa Binh, Lao Cai, Lam Dong [Nguyen, 2007; Nguyen, Pham, 2011].

29. Ropalidia cyathiformis (Fabricius, 1804)

Eumenes cyathifornis Fabricius, 1804: 289; Ropalidia cyathiformis van der Vecht, 1941: 104

DISTRIBUTION. Nepal; India; Sri Lanka; Myanmar; Malaysia: Malay Peninsula; Indonesia: Java, Sulawesi, Sum- 
ba; Philippines; Vietnam: Binh Dinh, Hanoi, Ha Giang, Hoa Binh, Thai Nguyen, Vinh Phuc [Nguyen et al., 2005, 2006b; Nguyen, 2007].

NOTE. New record within Hanoi $\left(10^{\top} 3\right.$ \% $9+$ - Nghia Do, Cau Giay, $21^{\circ} 03^{\prime} \mathrm{N} 105^{\circ} 50^{\prime} \mathrm{E}, \mathrm{h}=9 \mathrm{~m}, 29 . \mathrm{V} .2012$, PP).

30. Ropalidia fasciata (Fabricius, 1804)

Eumenes fasciata Fabricius, 1804: 290; Polistes bioculata Fabricius 1804: 278; Icaria picta de Saussure, 1854: 238; Icaria maculifrons Cameron, 1903: 172; Icaria intermedia Cameron, 1905 70; Ropalidia picta van der Vecht, 1941: 104, 111, 145; Ropalidia fasciata van der Vecht, 1959a: 245; 1962: 23.

DISTRIBUTION. Nepal; India; Myanmar; Thailand; Malaysia: Malay Peninsula; Indonesia: Sumatra, Nias, Bangka, Java, Karimon Djawa, Bali, Flores; South China; Palawan; Taiwan; Ryukyu Is.; Vietnam: Ba Ria-Vung Tau, Ca Mau, Dong Nai, Hai Phong, Kien Giang, Lam Dong, Lang Son, Nghe An, Sai Gon, Son La [van der Vecht, 1962; Nguyen et al., 2005, 2006b; Nguyen, 2007; Nguyen, Pham, 2011].

\section{Ropalidia flavopicta (F. Smith, 1857)}

Icaria flavopicta F. Smith, 1857: 99; Ropalidia flavopicta Bequaert, 1918: 246; Ropalidia flavopicta flavopicta van der Vecht, 1962: 42, 48, 49, 51

DISTRIBUTION. Hong Kong; Malaysia: Malay Peninsula; Indonesia: Sumatra; Vietnam: Ha Tinh, Gia Lai, Lam Dong, Quang Nam, Son La, Thai Nguyen [Kojima, 1996; Kojima, Carpenter, 1997; Nguyen et al., 2005, 2006b; Nguyen, 2007]

\section{Ropalidia hongkongensis (de Saussure, 1854)}

Icaria hongkongensis de Saussure, 1854: 239; Ropalidia hongkongensis hongkongensis; Lee, 1982. — Das et Gupta, 1984 (1983): 418; 1989: 111, 132; Ropalidia hongkongensis juncta van der Vecht, 1941: 141.

DISTRIBUTION. South China; Hong Kong; India: Meghayala; Myanmar; Indonesia: Bangka, Java; Vietnam: Ha Giang, Hai Phong, Lang Son, Nghe An, Son La, Thai Nguyen, Vinh Phuc, Yen Bai [Nguyen et al., 2005, 2006b; Nguyen, 2007; Nguyen, Pham, 2011], Quang Ninh.

NOTE. New record for Quang Ninh Province (1으 Phuong Dong, Uong Bi, $21^{\circ} 04^{\prime} \mathrm{N} 106^{\circ} 45^{\prime} \mathrm{E}, \mathrm{h}=123 \mathrm{~m}$, 26.VI.2013, PP).

\section{Ropalidia magnanima van der Vecht, 1941}

Ropalidia magnanima albitarsis van der Vecht, 1941: 125; 1962: 9; Ropalidia magnanima anthracina Das et Gupta, 1989: 110, 115-116; Ropalidia magnanima magnanima van der Vecht, 1941: 109, 125; 1962: 9

DISTRIBUTION. Myanmar; Malaysia: Malay Peninsula; Vietnam: Hoa Binh, Kien Giang, Sai Gon, Tay Ninh [van der Vecht, 1941; Kojima, Carpenter, 1997; Nguyen et al., 2005, 2006b; Nguyen, 2007; Nguyen, Pham, 2011], Quang Ninh.

NOTE. New record for the Provinces Quang Ninh $(290$ - Phuong Dong, Uong Bi, $21^{\circ} 04^{\prime} \mathrm{N} 106^{\circ} 45^{\prime} \mathrm{E}, \mathrm{h}=123 \mathrm{~m}$, 26-27.VI.2013, PP) and Hoa Binh (3우 — Mai Chau town, $20^{\circ} 39^{\prime} \mathrm{N} 105^{\circ} 04^{\prime} \mathrm{E}, \mathrm{h}=287 \mathrm{~m}, \mathrm{PP}$, nest No 201201).

\section{Ropalidia malayana (Cameron, 1903)}

Icaria malayana Cameron, 1903: 171; Icaria parvimaculata Cameron, 1907: 25; Ropalidia delicata Dover, 1931 (1930): 257; Ropalidia malayana van der Vecht, 1941: 104, 112, 174; 1962: 34

DISTRIBUTION. Malaysia: Malay Peninsula; Indonesia: Sumatra; Sunda Shelf; Borneo; Vietnam: Lam Dong [Nguyen et al., 2005, 2006b; Nguyen, 2007].
35. Ropalidia marginata

(Lepeletier de Saint Fargeau, 1836)

Vespa ferruginea Fabricius, 1793: 280; Epipona marginata Lepeletier de Saint Fargeau, 1836: 541; Ropalidia marginata marginata van der Vecht, 1941: 109, 117.

DISTRIBUTION. Pakistan; India; Sri Lanka; Vietnam: Ca Mau, Binh Phuoc, Dak Lak, Kien Giang, Ninh Thuan, Son La, Tay Ninh [Nguyen et al., 2005, 2006b; Nguyen, 2007].

\section{Ropalidia mathematica (F. Smith, 1860)}

Polybia mathematica F. Smith, 1860(1861): 90; Icaria nigroplagiata Cameron, 1900: 498; Ropalidia mathematica binotata van der Vecht, 1941: 131.

DISTRIBUTION. Indonesia: Sulawesi, Lombok, Flores, Java; Vietnam: Ha Giang, Hai Phong, Hanoi, Hoa Binh, Ha Tinh, Nghe An, Phu Tho, Quang Tri, Vinh Phuc [Nguyen et al., 2005, 2006b; Nguyen, 2007; Nguyen, Pham, 2011].

\section{Ropalidia modesta (F. Smith, 1858)}

Icaria modesta F. Smith, 1858: 115; Icaria fulvipennis Gribodo, 1892(1891): 245; Icaria ungulata Bingham, 1897: 387, 391; Ropalidia modesta van der Vecht, 1941: 104, 112, 187.

DISTRIBUTION. Myanmar; Thailand; Malaysia: Malay Peninsula; Indonesia: Sumatra; Java; Vietnam: Dong Nai, Tay Ninh [Nguyen et al., 2006b; Nguyen, 2007].

\section{Ropalidia nigrita Das et Gupta, 1989}

Ropalidia nigrita Das et Gupta, 1989: 111, 130.

DISTRIBUTION. India: Manipur; Vietnam: Hoa Binh [Nguyen et al., 2006b; Nguyen, 2007; Nguyen, Pham, 2011].

\section{Ropalidia ornaticeps (Cameron, 1900)}

Icaria ornaticeps Cameron, 1900: 496; Ropalidia flavopicta ornaticeps van der Vecht, 1962: 49.

DISTRIBUTION. India; Myanmar; Thailand; Cambodia; Malaysia: Malay Peninsula; Vietnam: Ba Ria — Vung Tau, Dak Lak, Dong Nai, Ha Giang, Ha Tinh, Hoa Binh, Lam Dong, Nghe An, Phan Rang, Phu Tho, Quang Binh, Quang Tri, Son La [Kojima, 1996; Kojima, Carpenter, 1997; Nguyen et al., 2005, 2006b; Nguyen, 2007].

\section{Ropalidia rufocollaris (Cameron, 1900)}

Icaria rufocollaris Cameron, 1900: 497; Ropalidia rufocollaris atrata van der Vecht, 1941: 139

DISTRIBUTION. India: Meghalaya, Assam, Sikkim, Tipura, Uttar Pradesh, West Bengal; China: Tibet; Myanmar; Thailand; Vietnam: Dien Bien, Hanoi, Ha Giang, Nghe An, Son La [Nguyen et al., 2005, 2006b; Nguyen, 2007; Nguyen, Pham, 2011].

\section{Ropalidia rufoplagiata (Cameron, 1905)}

Icaria rufoplagiata Cameron, 1905b: 71; Ropalidia rufoplagiata van der Vecht, 1941: 149.

DISTRIBUTION. India; Myanmar; Thailand; Malaysia: Peninsular Malaysia; Indonesia: Sumatra, Bangka, Java, Sumbawa; Timor; Vietnam: Hai Phong [Nguyen et al., 2006b; Nguyen, 2007].

\section{Ropalidia stigma (F. Smith, 1858)}

Polybia stigma Smith, 1858: 114; Ropalidia stigma stigma van der Vecht, 1941: 110, 126; 1962: 16

DISTRIBUTION. India; Sri Lanka; Myanmar; Thailand; Malaysia: Malay Peninsula; Indonesia: Sumatra, Java, Bali; Philippines; Vietnam: Binh Thuan, Ca Mau, Dong Nai, Hanoi, Ha Giang, Ha Tinh, Hoa Binh, Dak Lak, Kien 
Giang, Kon Tum, Lai Chau, Lang Son, Lam Dong, Ninh Binh, Nghe An, Phu Tho, Quang Tri, Son La, Quang nam, Tay Ninh, Thua Thien Hue, Vinh Phuc [Kojima, Carpenter, 1997; Nguyen et al., 2006b; Nguyen, 2007; Nguyen, Pham, 2011], Quang Ninh.

NOTE. New record for Quang Ninh Province (2o+ Phuong Dong, Uong $\mathrm{Bi}, 21^{\circ} 04^{\prime} \mathrm{N} 106^{\circ} 45^{\prime} \mathrm{E}, \mathrm{h}=123 \mathrm{~m}$, 26.VI.2013, PP; 3 우 — Yen Tu NP, Uong Bi, $21^{\circ} 02^{\prime} \mathrm{N}$ $106^{\circ} 36^{\prime} \mathrm{E}, \mathrm{h}=257 \mathrm{~m}, 27$. VI.2013, PP).

\section{Ropalidia sumatrae (Weber, 1801)}

Vespa sumatrae Weber, 1801: 103; Vespa mutillata Illiger, 1802: 189; Polistes pubescens Fabricius, 1804: 279; Eumenes formicaria Fabricius, 1804: 288; Anthreneida coronata White, 1841: 321; Icaria speciosa de Saussure, 1855b: 374; Icaria rufinoda Cameron, 1904: 121; Ropalidia speciosa Dover, 1929: 47; Ropalidia sumatrae van der Vecht, 1941: 104

DISTRIBUTION. Myanmar; Thailand; Malaysia: Malay Peninsula; Singapore; China: Yunnan; Indonesia: Bangka; Sumatra; Vietnam [Kojima, Carpenter, 1997].

NOTE. This species was recorded from Vietnam without exact locality [Kojima, Carpenter, 1997a].

\section{Ropalidia taiwana Sonan, 1935}

Ropalidia taiwana Sonan, 1935: 199, 201; Ropalidia taiwana var. koshunensis Sonan, 1935: 199, 202; Ropalidia formosana Kuo, 1987: 84.

DISTRIBUTION. North Myanmar; South China; Taiwan; Vietnam: Hoa Binh, Lam Dong, Lao Cai, Thua Thien Hue, Vinh Phuc [Nguyen et al., 2005, 2006b; Nguyen, 2007; Nguyen, Pham, 2011]

\section{Ropalidia thailandia Gusenleitner, 1994}

Ropalidia thailandia Gusenleitner, 1994: 325-329.

DISTRIBUTION. Thailand: Phang Nga; Vietnam: Lam Dong [Kojima, 1997; Nguyen et al., 2005, 2006b; Nguyen, 2007].

46. Ropalidia vietnama Gusenleitner, 1996

Ropalidia vietnama Gusenleitner, 1996: 15-17.

DISTRIBUTION. Vietnam: Lam Dong [Gusenleitner, 1996; Kojima, Carpenter, 1997; Nguyen et al., 2005, 2006b; Nguyen, 2007].

\section{Parapolybia de Saussure, 1854}

47. Parapolybia indica (de Saussure, 1854)

Polybia indica de Saussure, 1854: 207; Parapolybia indica von Schulthess, 1913: 153-154; Parapolybia takasagona Sonan, 1944: $342,344$.

DISTRIBUTION. India; Myanmar; Borneo; China; Taiwan; Korea; Japan; Vietnam: Dien Bien, Hanoi, Ha Giang, Hai Phong, Ha Tinh, Hoa Binh, Gia Lai, Lai Chau, Ninh Binh, Nghe An, Phu Tho, Thua Thien Hue, Vinh Phuc [Nguyen et al., 2005; Nguyen, 2007; Nguyen, Pham, 2011].

\section{Parapolybia nodosa van der Vecht, 1966}

Parapolybia nodosa van der Vecht, 1966: 25, 39

DISTRIBUTION. India; Nepal; Myanmar; Thailand; China; Taiwan; Vietnam: Hanoi, Ha Tinh, Hai Phong, Hoa Binh, Son La, Vinh Phu [Nguyen et al., 2005; Nguyen, 2007; Nguyen, Pham, 2011], Quang Ninh.

NOTE. New record for Quang Ninh Province $\left(2+0^{7}-\right.$ Phuong Dong, Uong $\mathrm{Bi}, 21^{\circ} 04^{\prime} \mathrm{N} 106^{\circ} 45^{\prime} \mathrm{E}, \mathrm{h}=123 \mathrm{~m}$, 26.VI.2013, PP; $10^{\top}-$ Yen Tu NP, Uong Bi, $21^{\circ} 02^{\prime} \mathrm{N}$ $106^{\circ} 36^{\prime} \mathrm{E}, \mathrm{h}=257 \mathrm{~m}, 27$. VI.2013, PP).

\section{Parapolybia varia (Fabricius, 1787)}

Vespa varia Fabricius, 1787: 293; Polybia orientalis de Saussure, 1854: 208; Polybia artifex F. Smith, 1860 (1861): 90; Icaria quadrimaculata Cameron, 1900: 496; Icaria carinata Cameron, 1900: 499; Icaria wroughtoni Cameron, 1900: 500; Icaria singapurensis Cameron, 1904: 120; Icaria annulipes Cameron, 1913: 115; Stelopolybia disticha du Buysson, 1913: 298; Parapolybia orientalis von Schulthess, 1913: 153, 155; Parapolybia varia Liu, 1936: 205,348

DISTRIBUTION. India; Nepal; Myanmar; Thailand; Malaysia: Malay Peninsula; Singapore; Indonesia: Sulawesi, Sumbawa, Sumba; Philippines; Riouw-Archipelago (= Kepulauan Riau); China; Korea; Japan; Vietnam: Cao Bang, Dien Bien, Gia Lai, Ha Giang, Hai Phong, Hanoi, Ha Tinh, Hoa Binh, Lang Son, Lai Chau, Lam Dong, Lao Cai, Nghe An, Quang Binh, Son La, Thanh Hoa, Thua Thien Hue, Vinh Phuc [Nguyen et al., 2005; Nguyen, 2007; Nguyen, Pham, 2011].

Polybioides du Buysson, 1913

50. Polybioides gracilis van der Vecht, 1966

Polybioides gracilis van der Vecht, 1966: 13, 19

DISTRIBUTION. Myanmar; Malaysia: Malay Peninsula; Vietnam: Kontum [van der Vecht, 1966; Kojima, Carpenter, 1997; Nguyen et al., 2005; Nguyen, 2007].

Subfamily Vespinae

Provespa Ashmead, 1903

1. Provespa barthelemyi (du Buysson, 1905)

Vespa barthelemyi du Buysson, 1905 (1904): 492, 618.

DISTRIBUTION. India: Sikkim, Assam; Bhutan; China: Yunnan, Guangxi; Myanmar; Thailand; Laos: Pnompenh; Cambodia; Malaysia: Peninsular Malaysia; Vietnam: Bac Can, Gia Lai, Nghe An, Phu Tho, Quang Tri, Quang Nam, Vinh Phuc [Carpenter, Kojima, 1997b; Nguyen, Carpenter, 2002; Nguyen et al., 2005, 2006a; Nguyen, 2007].

\section{Provespa nocturna van der Vecht, 1935}

Provespa nocturna van der Vecht, 1935: 41.

DISTRIBUTION. Malaysia: Peninsular Malaysia, Sarawak; Indonesia: Sumatra, Bangka, Kalimantan; Vietnam: Hoa Binh [van der Vecht, 1957; Carpenter, Kojima, 1997b; Nguyen, Carpenter, 2002; Nguyen et al., 2005, 2006a; Nguyen, 2007].

Van der Vecht [1957] recorded this species from "Indochina:... Tonkin, rég. de Hoa Binh, 1929" based on a worker. Lately, Nguyen and Carpenter [2002], Nguyen et al. [2006a], and Nguyen [2007] recorded the species for Vietnam, but did not bring any examined specimen out.

Vespa Linnaeus, 1758

\section{Vespa affinis (Linnaeus, 1764)}

Apis affinis Linnaeus, 1764: 417; Vespa alduini Guérin-Méneville, 1831: 9; 1838: 264; Vespa cincta var. picea du Buysson, 1905a (1904): 488, 537; Vespa indosinensis Pérez, 1910: 8; Vespa fonnosana Sonan, 1927: 125; Vespa affinis continentalis Bequaert, 1936: 346, 350; Vespa affinis var. hainanensis Bequaert, 1936: 347, 349; Vespa affinis nigriventris van der Vecht, 1957: 28-29; Vespa affinis archboldi van der Vecht, 1957: 28, 32;

DISTRIBUTION. India; Sri Lanka; Myanmar; China; Taiwan; Japan: Ryukyu Is.; Thailand; Laos; Malaysia: Peninsular Malaysia, Sabah, Sarawak; Singapore; Papua New Guinea, including New Britain and New Ireland; Philippines: Palawan, Dumaran, Labuan, Mindanao, Samar, Luzon; Indonesia: Sumatra, Nias, Bangka, Java, Kalimantan, Sulawesi, 
Talaud Islands, Buton, Selayar, Sunda Is., Timor, Moluccas, Papua; Vietnam: Bac Giang, Dien Bien, Hanoi, Hai Phong, Ha Tinh, Hoa Binh, Gia Lai, Kien Giang, Lang Son, Lao Cai, Ninh Binh, Nghe An, Phu Tho, Phu Yen, Quang Ninh, Quang Tri, Quang Binh, Quang Nam, Son La, Thua Thien Hue, Thai Nguyen, Vinh Phuc [Carpenter, Kojima, 1997b; Nguyen, Carpenter, 2002; Nguyen et al., 2005, 2006a; Nguyen, 2007; Nguyen, Pham, 2011], Thai Binh.

NOTE. New record for Thai Binh Province (1ㄴ - Hong Minh, Hung Ha, $20^{\circ} 35^{\prime} \mathrm{N} 106^{\circ} 08^{\prime} \mathrm{E}, \mathrm{h}=3 \mathrm{~m}, 23$.VI.2013, PP; 1 우 - Hung Nhan, Hung Ha, $20^{\circ} 31^{\prime} \mathrm{N} 106^{\circ} 11^{\prime} \mathrm{E}, \mathrm{h}=2 \mathrm{~m}$ 25.VI.2013, PP).

\section{Vespa analis Fabricius, 1775}

Vespa analis Fabricius, 1775: 363; Vespa analis var. tenebrosa du Buysson, 1905a (1904): 516.

DISTRIBUTION. India; Nepal; China; Myanmar; Thailand; Laos; Malaysia: Peninsular Malaysia; Singapore; Taiwan; Korea; Japan: Ryukyu Is.; Russia: Amur, Primor'ye; Indonesia: Sumatra, Bangka, Sunda Islands, Java, Bawean, Bali, Madura, Lombok; Vietnam: Cao Bang, Dien Bien, Hoa Binh, Lai Chau, Lao Cai, Quang Nam, Quang Tri, Son La, Thai Nguyen, Thanh Hoa, Thua Thien Hue, Vinh Phuc [Nguyen, Carpenter, 2002; Nguyen et al., 2005, 2006a; Nguyen, 2007; Nguyen, Pham, 2011].

\section{Vespa auraria F. Smith, 1852}

Vespa auraria F. Smith, 1852: 46; Vespa velutina pruthii van der Vecht, 1959c: 228.

DISTRIBUTION. Afghanistan; Pakistan; India: Kashmir, Himachal Pradesh, Punjab, Uttar Pradesh, West Bengal, Meghalaya, Sikkim, Assam; Nepal; Myanmar; Thailand; Laos; China: Yunnan; Malaysia: Peninsular Malaysia; Yemen; Vietnam: Hanoi, Ha Giang, Ninh Binh, Lam Dong, Sõn La, Vinh Phuc [Nguyen, Carpenter, 2002; Nguyen et al., 2005].

\section{Vespa basalis F. Smith, 1852}

Vespa basalis F. Smith, 1852: 46; Vespa obliterata F. Smith, 1852: 47.

DISTRIBUTION. Pakistan; India: Himachal Pradesh, West Bengal, Meghalaya, Sikkim, Assam; Nepal; China: Sichuan, Yunnan, Fujian; Taiwan; Myanmar; Thailand; Laos; Indonesia: Sumatra; Vietnam: Bac Can, Ha Giang, Hoa Binh, Quang Nam, Thai Nguyen, Thanh Hoa, Thua Thien Hue, Vinh Phuc [Carpenter, Kojima, 1997b; Nguyen, Carpenter, 2002; Nguyen et al., 2005, 2006a; Nguyen, 2007].

\section{Vespa bicolor Fabricius, 1787}

Vespa bicolor Fabricius, 1787: 288; Vespa lutea Coquebert, 1804: 94; Vespa auraria var. citriventris du Buysson, 1905 (1904): 552.

DISTRIBUTION. India: Uttar Pradesh, West Bengal, Sikkim, Assam, Meghalaya; Bhutan; Nepal; China: Sichuan, Yunnan, Guangxi, Jiangxi, Fujian; Xianggang; Myanmar; Thailand; Laos; Cambodia; Vietnam: Bac Can, Hanoi, Ha Giang, Ha Tinh, Lai Chau, Lam Dong, Lao Cai, Nghe An, Phu Tho, Quang Ninh, Quang Nam, Quang Binh, Thua Thien Hue, Thai Nguyen, Vinh Phuc [Carpenter, Kojima, 1997b; Nguyen, Carpenter, 2002; Nguyen et al., 2005, 2006a; Nguyen, 2007].

\section{8. ?Vespa binghami du Buysson, 1905}

Vespa binghami du Buysson, 1905(1904): 488, 523; Vespa suprunenkoi Birula, 1925(1924): 89, 92.

DISTRIBUTION. India: West Bengal, Assam, Meghalaya; Bhutan; Myanmar; Thailand; Laos; China: Xizang, Sichuan, Yunnan, Jiangsu, Shanghai; Russia: Primor'ye, Sa- khalin; Korea; Vietnam [Archer, 1989; Carpenter, Kojima, 1997b; Nguyen, Carpenter, 2002; Nguyen et al., 2005, 2006a; Nguyen, 2007].

Archer [1989] recorded this species from North Vietnam, but did not show the exact locality. In his next studies [Archer, 1999] lately, he did not mention the present of the species in Vietnam. According to my ideas, this may be a mistake in his papers [1989 or 1999]. Nguyen's studies on the subfamily Vespinae and the social wasp in Vietnam [Nguyen, 2002, 2005, 2006a, 2007] also mentioned the presence of this species in Vietnam, but that was not documented. Occurring of the species is thus ambiguous. In this list, however, we still include the species occurring in Vietnam but put a question remark in front of the name of the species.

\section{Vespa ducalis F. Smith, 1852}

Vespa ducalis F. Smith, 1852: 39; Vespa ducalis var. pulchra du Buysson, 1905 (1904): 519; Vespa matsumurai Sonan, 1935: 370; Vespa esakii Sonan, 1935: 371; Vespa tropica loochooensis Bequaert, 1936: 336, 343; Vespa tropica pseudosoror van der Vecht, 1959c: 224

DISTRIBUTION. India: Sikkim, Meghalaya; Nepal; Myanmar; Thailand; China: Gansu, Sichuan, Hainan, Guangdong, Fujian, Hunan, Shanghai, Jiangsu, Liaoning; Taiwan; Russia: Primor'ye; Korea; Japan: Ryukyu Is.; Vietnam: Hanoi, Ha Giang, Hoa Binh, Lao Cai, Ninh Binh, Phu Tho, Quang Tri, Quang Nam, Son La, Thanh Hoa, Ha Tinh, Thua Thien Hue, Vinh Phuc [van der Vecht, 1959ñ; Carpenter, Kojima, 1997b; Nguyen, Carpenter, 2002; Nguyen et al., 2005, 2006a; Nguyen, 2007; Nguyen, Pham, 2011].

\section{Vespa mandarinia F. Smith, 1852}

Vespa mandarinia F. Smith, 1852: 38; Vespa magnifica Smith, 1852: 45; Vespa japonica Radoszkowski, 1857: 410; Vespa bellona Smith, 1871: 248; Vespa magnifica var. latilineata Cameron, 1903: 278; Vespa magnifica var. nobilis Sonan, 1929: 137, 140; Vespa magnifica sonani Matsumura, 1930: 1, 5.

DISTRIBUTION. India: Himachal Pradesh, Uttar Pradesh, West Bengal, Sikkim, Assam, Meghalaya; Sri Lanka; Nepal; Bhutan; Myanmar; Thailand; Laos; Malaysia: Peninsular Malaysia; China: Sichuan, Yunnan, Xianggang, Jiangxi, Fujian, Rubei, Shanghai, Jiangsu; Taiwan; Russia: Primor'ye; Korea; Japan: Yakushima, Tanegashima; Vietnam: Dien Bien, Hoa Binh, Son La [Nguyen, Carpenter, 2002; Nguyen et al., 2005, 2006a; Nguyen, 2007].

\section{Vespa mocsaryana du Buysson, 1905}

Vespa mocsaryana du Buysson, 1905a (1904): 490, 537.

DISTRIBUTION. India: Meghalaya, Sikkim, Assam; China: Sichuan, Fujian, Anhui; Myanmar; Thailand; Laos; Malaysia: Peninsular Malaysia; Indonesia: Sumatra; Vietnam: Dien Bien, Hoa Binh, Hanoi, Kon Tum, Ninh Binh, Nghe An, Phu Tho, Quang Nam, Thai Nguyen, Thua Thien Hue, Vinh Phuc [van der Vecht, 1957; Carpenter, Kojima, 1997b; Nguyen, Carpenter, 2002; Nguyen et al., 2005, 2006a; Nguyen, 2007; Nguyen, Pham, 2011].

\section{Vespa soror du Buysson, 1905}

Vespa ducalis var. soror du Buysson, 1905 (1904): 490, 519.

DISTRIBUTION. India; China: Yunnan, Rainan, Fujian; Thailand; Laos; Vietnam: Cao Bang, Bac Can, Ha Tinh, Hoa Binh, Lam Dong, Ninh Binh,oaHoaHosaaa Nghe An, Phu Tho, Quang Ninh, Quang Nam, Quang Tri, Son La, Thai Nguyen, Thua Thien Hue, Vinh Phuc [Carpenter, Kojima, 1997b; Nguyen, Carpenter, 2002; Nguyen et al., 2005, 2006a; Nguyen, 2007; Nguyen, Pham, 2011]. 


\section{Vespa tropica (Linnaeus, 1758)}

Sphex tropica Linnaeus, 1758: 571; Vespa unicolor F. Smith, 1863 (1864): 44; Vespa affinis var. trisignata Pérez, 1910: 8; Vespa tropica leefmansi van der Vecht, 1957: 19; Vespa tropica trimeres van der Vecht, 1957: 19, 22; Vespa tropica cebuana Kojima et Reyes, 1984: 260.

DISTRIBUTION. Afghanistan; Pakistan; India; Sri Lanka; Nepal; Bhutan; China: Yunnan, Xianggang, Fujian; Myanmar; Thailand; Laos; Cambodia; Malaysia: Peninsular Malaysia, Sarawak; Papua New Guinea, including New Britain; Philippines; Indonesia: Anambas Islands, Sumatra, Nias, Batu, Enggano, Bangka, Belitung, Sunda Islands, Java, Bawean, Madura, Kangean Islands, Bali, Lombok, Sumbawa, Sumba, Komodo, Flores, Wetar, Kalimantan, Sulawesi, Selayar, Sula Is., Moluccas, Papua; Timor; Vietnam: Ca Mau, Bac Can, Dien Bien, Gia Lai, Hanoi, Hoa Binh, Ha Nam, Kien Giang, Lai Chau, Nghe An, Phu Tho, Quang Ninh, Quang Tri, Son La, Thanh Hoa, Thua Thien Hue, Thai Nguyen, Vinh Phuc [Carpenter, Kojima, 1997b; Nguyen, Carpenter, 2002; Nguyen et al., 2005, 2006a; Nguyen, 2007; Nguyen, Pham, 2011].

\section{Vespa velutina}

Lepeletier de Saint Fargeau, 1836

Vespa velutina "De Haan" Lepeletier de Saint Fargeau, 1836: 507; Vespa auraria F. Smith, 1852: 46; Vespa fruhstorferi Stadelmann, 1894: 89; Vespa velutina var. ardens du Buysson, 1905a (1904): 550; Vespa velutina var. celebensis Pérez, 1910: 12; Vespa velutina sumbana van der Vecht, 1957: 34, 40; Vespa velutina karnyi van der Vecht, 1957: 35, 38;

DISTRIBUTION. India: West Bengal, Sikkim, Assam; Bhutan; China: Si-chuan, Jiangxi, Fujian, Hubei, Zhejiang, Xianggang; Taiwan; Myanmar; Thailand; Laos; Malaysia: Peninsular Malaysia; Indonesia: Sumatra, Java, Bali, Lombok, Sumbawa, Komodo, Sumba, Flores, Timor, Sulawesi; Vietnam: Cao Bang, Dien Bien, Hanoi, Ha Giang, Ha Tinh, Hoa Binh, Lai Chau, Lao Cai, Lam Dong, Nghe An, Phu Tho, Quang Tri, Quang Binh, Quang Nam, Son La, Thua Thien Hue, Thai Nguyen, Vinh Phuc [Carpenter, Kojima, 1997b; Nguyen, Carpenter, 2002; Nguyen et al., 2006a; Nguyen, 2007; Nguyen, Pham, 2011], Quang Ninh.

NOTE. New record for Quang Ninh Province $(1+$ - Yen $\mathrm{Tu}$ NP, Uong Bi, $21^{\circ} 02^{\prime} \mathrm{N} 106^{\circ} 36^{\prime} \mathrm{E}, \mathrm{h}=257 \mathrm{~m}, 27 . \mathrm{VI} .2013$, PP).

\section{Vespula Thomson, 1869}

15. Vespula koreensis (Radoszkowski, 1887)

Vespa koreensis Radoszkowski, 1887: 432; Vespa germanica var. stizoides du Buysson, 1905 (1904): 494, 615; ?Vespa orbata var. aurulenta du Buysson, 1905 (1904): 579; Pseudovespa birulai Gussakovskii, 1932: 52; Vespula koreensis salebrosa Archer, 1982: 261, 267; Vespula hainanensis Lee, 1986: 201, 202, 206.

DISTRIBUTION. India: Sikkim, Meghalaya; Thailand; Laos; China: Sichuan, Yunnan, Hainan, Jiangxi, Fujian, Hunan, Anhui, Zhejiang, Hebei, Beijing, Liaoning, Heilongjiang; Korea; Russia: Amur, Primor'ye; Vietnam: Phu Tho, Quang Tri, Thai Nguyen [Nguyen et al., 2006a; Nguyen, 2007].

\section{Vespula orbata (du Buysson, 1902)}

Vespa orbata du Buysson, 1902: 140; Vespa minuta Dover, 1925 (1924): 304; Vespula orbata: van der Vecht, 1959c: 217.

DISTRIBUTION. India: Uttar Pradesh, West Bengal, Assam; Nepal; Myanmar; Vietnam: Ha Giang, Ha Tinh, Lao Cai, Quang Nam [Nguyen, Carpenter, 2002; Nguyen et al., 2005, 2006a; Nguyen, 2007].
ACKNOWLEDGMENTS. We are very grateful to Prof. Khuat Dang Long (IEBR) for providing some specimens for study, to PhD. student Hari Nugroho (Museum Zoologicum Bogoriense, Research Center for Biology, Indonesian Institute of Sciences, Indonesia) for providing some data for study, and also to Mr. Khuc Van Linh for the help with collecting specimens.

\section{References}

Archer M.E. 1989. A key to the world species of the Vespinae (Hymenoptera) // Res. Mg. Coll. Ripon \& York St. John. Vol.2. No.1. P.1-41.

Archer M.E. 1999. Taxonomy, distribution and nesting biology of Vespa binghami, V. basalis, $V$. variabilis, V. fervida, V. luctuosa, V. multimaculata, and V. bellicosa (Hymenoptera, Vespinae) // Enomol. Mon. Mag. Vol.135. P.43-50.

Bequaert J.C. 1928. A study of certain types of diplopterous wasps in the col-lection of the British Museum // Ann. Mag. Nat. Hist. Vol.2. No.10. P.138-176.

Carpenter J.M. 1996. Distributional Checklist of Species of the Genus Polistes (Hymenoptera: Vespidae; Polistinae, Polistini) // Am. Mus. Novit. Vol.3188. P.1-39.

Carpenter J.M., Kojima J. 1997. Checklist of the species in the Subfamily Stenogastrinae (Hymenoptera: Vespidae) // J. New York Entomol. Soc. Vol.104. No.1-2. P.21-36.

Carpenter J.M., Kojima J. 1997b. Checklist of the species in the subfamily Vespinae (Insecta: Hymenoptera: Vespidae) // Nat. Hist. Bull. Ibaraki Univ. Vol.1. P.51-92.

Carpenter J.M., Starr C.K. 2000. A new genus of hover wasps from Southeast Asia (Hymenoptera: Vespidae; Stenogastrinae) // Am. Mus. Novit. Vol. 3291. P.1-12.

Dang T.H., Nguyen T.P.L., Kojima J. 2012. Taxonomic notes on the genus Euodynerus Dalla Torre (Hymenoptera: Vespidae: Eumeninae) from Northern Vietnam // J. Biol. Vol.34. No.4. P.427-431.

Giordani Soika A. 1941. Studi sui Vespidi Solitari // Boll. Soc. Veneziana Stor. Nat. Vol.2. P.130-279.

Giordani Soika A. 1973. Notulae vespidologicae XXXV. Descrizione di nuovi Eumenidi // Boll. Mus. Civ. Stor. Nat. Venezia. Vol. 24. P.97-131.

Giordani Soika A. 1982 (1981). Revisione delle specie orientali del genere Antepipona Sauss. (Hym. Vespoidea) // Boll. Mus. Civ. Stor. Nat. Venezia. Vol.32. P.205-257.

Giordani Soika A. 1986. Eumenidi di Okinawa e delle Filippine Raccolti da J. Kojima // Boll. Mus. Civ. Stor. Nat. Venezia. Vol.35. P.67-89.

Giordani Soika A. 1993. Di Alcuni Nuovi Eumenidi della Regione Orientale (Hym. Vespoidea) // Boll. Mus. Civ. Stor. Nat. Venezia. Vol.42. P.151-163.

Giordani Soika A. 1994. Ricerche sistematiche su alcuni generi di Eumenidi della Regione Orientale e della Papuasia (Hymenoptera, Vespoidea) // An. Mus. Civ. Stor. Nat. Giacomo Doria. Vol.90. P. $1-348$.

Gusenleitner J. 1996. Zwei neue Arten der Gattung Ropalidia Guérin-Méneville aus der orientalischen Region (Hymenoptera, Vespidae) // Linzer Biol. Beitr. Vol.28. P.15-21.

Gusenleitner J. 2001. Beitrag zur Kenntnis von Faltenwespen der orientalischen Region (Hymenoptera, Vespidae, Eumenidae) // Linzer Biol. Beitr. Vol.33. No.2. P.655-662.

Gusenleitner J. 2010. Bemerkenswerte Faltenwespen-Funde aus der orientalischen Region Teil 5 (Hymenoptera: Vespidae, Eumeninae) // Linzer Biol. Beitr. Vol.42. No.1. P.695-709.

Gusenleitner J. 2012. Bemerkenswerte Faltenwespen-Funde aus der orientalischen Region Teil 6 (Hymenoptera: Vespidae, Eumeninae) // Linzer Biol. Beitr. Vol.44. No.2. P.1045-1052.

Gusenleitner J. 2013. Bemerkenswerte Faltenwespen-Funde aus der orientalischen Region Teil 7 (Hymenoptera: Vespidae, Eumeninae, Polistinae) // Linzer Biol. Beitr. Vol.45. No.1. P.121-132.

Kojima J. 1996. Taxonomy of the Ropalidia flavopicta-complex (Hymenoptera: Vespidae: Polistinae) // Zool. Med. Leiden. Vol.70. No.22. P.325-347. 
Kojima J. 1997. Subgeneric affiliation of Ropalidia thailandia Gusenleitner: a case showing ambiguity of the subgenera of Ropalidia Guérin- Méneville (Hymenoptera: Vespidae: Polistinae) // Zool. Med. Leiden. Vol.71. No.8. P.57-61.

Kojima J., Carpenter J.M. 1997. Catalog of Species in the Polistine Trible Ropalidiini (Hymenoptera: Vespidae) // Am. Mus. Novit. Vol.3199. P.1-96.

Nguyen T.P.L. 2007. Taxonomic revision and distribution pattern of social wasps (Hymenoptera: Vespidae) in Viet Nam // $\mathrm{PhD}$ Ibaraki University, Mito, Japan. 220pp.

Nguyen T.P.L., Carpenter J.M. 2002. Vespidae of Vietnam (Insecta: Hymenoptera) 1. Vespinae// J. New York Entomol. Soc. Vol.110. No.2. P.199-211.

Nguyen T.P.L., Carpenter J.M. 2013. Taxonomic notes on the species of the genus Malayepipona Giordani Soika (Hymenoptera: Vespidae: Eumeninae) from Northern Vietnam, with description of three new species // Raffles. Bul. Zool. Vol.61. No.2. P.727-734.

Nguyen T.P.L., Khuat D.L. 2004. [Study on the social wasps family Vespidae of Vietnam and three newly recorded species of the genus Eustenogaster van der Vecht, 1969 (Hymenoptera: Vespidae: Stenogastrinae) for Vietnam] // J. Biol. Vol.26. No.3A. P.38-42 [in Vietnamese, with English summary].

Nguyen T.P.L., Khuat D. L., Do V.L., Luong V.H. 2007. [Survey of bees and wasps (Hymenoptera) in Cuc Phuong National Park] // Proceedings of the $2^{\text {nd }}$ National Scientific Conference on Ecology and Biological Resources. P.415-419 [in Vietnamese, with English summary].

Nguyen T.P.L., Kojima J. 2013. Distribution and nests of paper wasps of Polistes (Polistella) in northeastern Vietnam, with description of a new species (Hymenoptera, Vespidae, Polistinae) // Zookeys. Vol.368. P.45-63.

Nguyen T.P.L., Kojima J., Saito F. 2011. Polistes (Polistella) wasps (Hymenoptera: Vespidae: Polistinae) from mountainous areas of northern Vietnam, with description of five new species // Zootaxa. Vol. 3060. P.1-30.

Nguyen T.P.L., Pham H.P. 2011. [Study on social wasps (Hymenoptera: Vespidae) of some natural reserves in Northwestern part] // Proceedings of the $7^{\text {th }}$ Vietnam National Conference on Entomology. P.848-851 [in Vietnamese, with English summary].

Nguyen T.P.L., Saito F., Kojima J., Carpenter J.M. 2005. [An annotated distributional checklist of social wasps (Hymenoptera: Vespidae) of Viet Nam] // Proceedings of the 1st National Workshop on Ecology and Biological Resources. Agricultural Pulishing House, Hanoi. P.129-137 [in Vietnamese, with English summary].

Nguyen T.P.L., Saito F., Kojima J., Carpenter J.M. 2006a. Vespidae of Vietnam (Insecta: Hymenoptera) 2. Taxonomic notes on Vespinae // Zool. Sci. Vol.23. No.1. P.95-104

Nguyen T.P.L., Saito F., Kojima J., Carpenter J.M. 2006b. Vespidae (Hymenoptera) of Viet Nam 3. Synoptic key to Vietnamese species of the polistine genus Ropalidia, with notes on taxonomy and distribution // Entomol. Sci. Vol.9. No.1. P.93-107.

Nugroho H., Kojima J., Carpenter J.M. 2011. Checklist of vespid species (Insecta: Hymenoptera: Vespidae) occurring in Indonesian Archipelago // Treubia. Vol.38. P.1-186.

Pickett K.M., Carpenter J.M. 2010. Simultaneous analysis and the origin of eusociality in the Vespidae (Insecta:Hymenoptera) // Arthropod Syst. \& Phyl. Vol.68. No.1. P.3-33.

Saito F. 2009. New species of hover wasps genus Eustenogaster (Insecta: Hymenoptera: Vespidae: Stenogastrinae) from Southeast Asia // Spec. Div. Vol.14. P.15-25.

Saito F., Nguyen T.P.L., Carpenter J.M., Kojima J. 2006. A new Eustenogaster species (Hymenoptera: Vespidae; Stenogastrinae), the first hover wasp known to overwinter on the nest // Am. Mus. Novit. Vol. 3534. P.1-11.

Saussure H. de. 1852. Études sur la Famille des Vespides 1. Monographie des Guêpes solitaires ou de la tribu des Euméniens // V. Masson, Paris and J. Kessmann, Genéve. P. 1-128.

Saussure H. de., André E., du Buysson R. 1904. [Hyménoptères recueillis par M.A.] // Mis. Pavie. Vol.3. P.188-203.

Srinivasan G., Girish K.P. 2010. New records of potter wasps (Hymenoptera: Vespidae: Eumeninae) from Arunachal Pradesh, India: five genera and ten species // J. Threat. Taxa. Vol.2. No.12. P.1313-1322.

Turillazzi S. 1991. The Stenogastrinae // The Social Biology of Wasps. Ithaca, New York: Cornell Univ. Press. P.74-98.

Vecht J. van der. 1941. The Indo-Australian species of the genus Ropalidia (= Icaria) (Hymenoptera, Vespidae) (first part) // Treubia. Vol.18. P.103-190.

Vecht J. van der. 1957. The Vespinae of the Indo-Malayan and Papuan areas (Hymenoptera, Vespidae) // Zool. Verh. Leiden. Vol.34. No.1. P.1-82.

Vecht J. van der. 1959a. On some Fabrician types of Indo-Australian Vespidae (Hymenoptera) // Arch. Néelandaises Zool. Vol.13. P.234-247.

Vecht J. van der. 1959b. On Eumenes arcuatus (Fabricius) and Some Allied Indo-Australian Wasps (Hymenoptera, Vespidae) // Zool. Verh. Leiden. Vol.41. P.1-73.

Vecht J. van der. 1959c. Notes on Oriental Vespinae, including some species from China and Japan (Hymenoptera, Vespidae) // Rijksmuseum Van Natuurlijke Historie te Leiden. Vol. 36. No.13. P.205-232.

Vecht J. van der. 1962. The Indo-Australian species of the genus Ropalidia (= Icaria) (Hymenoptera, Vespidae) (second part) // Zool. Verh. Leiden. Vol.57. P.1-72.

Vecht J. van der. 1963. Studies on Indo-Australian and East-Asiatic Eumenidae (Hymenoptera, Vespoidea) // Zool. Verh. Leiden. Vol.60. P.3-113.

Vecht J. van der. 1966. The east-Asiatic and Indo-Austalian species of Polybioides Buysson and Parapolybia Saussure (Hymenoptera, Vespidae) // Zool. Verh. Leiden. Vol.82. P.1-42.

Vecht J. van der. 1968. The geographic variation of Polistes (Megapolistes subg. $\mathrm{n}$.) rothneyi Cameron // Bijdragen tot de Dierkdunde. Vol.38. P.97-109.

Vecht J. van der, Carpenter J.M. 1990. A catalogue of the genera of the Vespidae (Hymenoptera) // Zool. Verh. Leiden. Vol.260. P.1-62. 